\title{
Artifacts Reduced Interpolation Method for Single-Sensor Imaging System
}

\author{
Long-Fei Wang \\ College of Telecommunications \& Information \\ Engineering, \\ Nanjing University of Posts and Telecommunications, \\ Nanjing, 210046, China \\ Email: wanglf9542@163.com
}

\author{
Si-Chen Zhou, Xin-Yi Peng \\ School of Oversea \\ Nanjing University of Posts and Telecommunications \\ Nanjing, 210046, China
}

\author{
Xiang-Dong Chen \\ School of Computer Science, \\ Nanjing University of Posts and Telecommunications, \\ Nanjing, 210046, China \\ Email: chenxd@njupt.edu.cn
}

\begin{abstract}
In this paper, we present a novel color image Demosaicking algorithm. The algorithm consists of two steps: an interpolation step and a refinement step. The missing green color information is first interpolated by using the color channel difference. In the refinement step, a local weighted directional interpolation method guided by the preinterpolated green channel is applied to refine the interpolation results along the determined interpolation direction. Lastly, post-processing is implemented to output the final Demosaicked full color image. Compared with the latest Demosaicking algorithms, experiments showed that the proposed method provides superior performance in terms of both objective and subjective image qualities.
\end{abstract}

Keywords-demosaicking; CFA interpolation; artifacts reduction

\section{INTRODUCTION}

When currently available digital still color cameras based on a single charge-coupled device (CCD) sensor capture a color pixel, only one part of the color information of the three color channels is captured. To reconstruct a full-color image, an interpolation process, commonly referred to CFA interpolation, is applied to estimate the other two missing color pixel values at each pixel position. This process is called CFA interpolation, or Demosaicking. Presently, the most common CFA in digital cameras uses a color arrangement based on the Bayer pattern [1, 2]. Fig. 1 shows a $7 \times 7$ window of Bayer CFA samples.

The color reproduction quality depends on the CFA templates and the employed Demosaicking algorithms. Various Demosaicking algorithms based on the Bayer pattern [3-13] have been proposed in the past decades. Recently developed methods include the successive approximation (SA) method by Li [4], the directional linear minimum mean square-error estimation (DL) method by Zhang and Wu [5], a least-squares luma-chroma demultiplexing (LSLCD) algorithm for Bayer Demosaicking by Dubois et al. [6], an effective Demosaicking method based on edge property (EDEP) by Chen and Chang [7], an adaptive filtering for color filter array Demosaicking (AFD) in the frequency domain proposed by Lian et al. [8], and the edge strength filter (ESF) based method by Pekkucuksen and Altunbasak [9]. A recent survey of Demosaicking methods can be found in [14]. Some of these methods exploit intra channel correlation (the color difference from green-to-green, red-to-red, and blue-to-blue) to determine the interpolation while others use the inter channel correlation (the color difference from green-to-red, green-to-blue, and red-to-blue). In the literature, methods use inter channel correlation have yielded better performance.

In this paper, we present a new color image Demosaicking algorithm. We first utilize the color difference between channels to populate the green (G) channel in advance, then a local weighted directional interpolation method is used to refine the green channel. The preinterpolated green channel is used to calculate the directional gradient since it supports more accurate edge information than conventional methods. These directional gradients in the working window are used to determine the interpolation direction. The pre-interpolation result is refined along the determined interpolation direction. Finally, we apply a postprocessing approach to remove interpolation artifacts by utilizing the directional weighted mean of neighboring color differences over channels.

The remainder of the paper is organized as follows. The proposed method including green channel interpolation and refinement, red (R) and blue (B) component interpolation, and overall plane refinements are described in Section II. We evaluate the Demosaicking performance of the conventional and the proposed methods in Section III. Finally, conclusions are made in Section IV.

\section{THE PROPOSED METHOD}

The green plane is usually reconstructed first because it contains twice as many samples as the red or blue planes. Thus, the green plane possesses most of the spatial information of the image to be interpolated and has great influence on the perceptual quality of the image. 
Furthermore, once the green plane is fully populated, the green plane can be used to guide the subsequent red and blue plane interpolation by making full and direct use of channel correlation.

\section{A. Green Channel Interpolation}

The green channel is interpolated in two steps. In the first step, we use the color differences $\kappa_{R}(=\mathrm{G}-\mathrm{R})$ and $\kappa_{B}(=\mathrm{G}-\mathrm{B})$. The green channel can be roughly interpolated in four directions: north $(\mathrm{N})$, south $(\mathrm{S})$, west $(\mathrm{W})$, and east $(\mathrm{E})$. The inter-channel correlation is exploited as the weighted factor to adjust the contribution of color differences among neighboring pixels. As illustrated in Fig. 1, the green pixel value at a location $R_{5}$ can be obtained by first calculating the $\kappa_{R}$ values of the four points surrounding $\mathrm{R}_{5}$, that is, $\mathrm{G}_{9}, \mathrm{G}_{16}$, $\mathrm{G}_{12}$, and $\mathrm{G}_{13}$. The $\kappa_{R}$ values of the four points are calculated by the following equations, respectively:

$$
\begin{aligned}
& \kappa_{R_{9}}=G_{9}-\frac{1}{2}\left(R_{2}+R_{5}\right) \\
& \kappa_{R_{16}}=G_{16}-\frac{1}{2}\left(R_{8}+R_{5}\right) \\
& \kappa_{R_{12}}=G_{12}-\frac{1}{2}\left(R_{4}+R_{5}\right) \\
& \kappa_{R_{18}}=G_{13}-\frac{1}{2}\left(R_{6}+R_{5}\right)
\end{aligned}
$$

Next, the absolute color gradients of the channels that measure the spatial correlations of the neighboring pixels $G_{9}$, $G_{16}, G_{12}$, and $G_{13}$ along the four directions are calculated as:

$$
\begin{aligned}
D_{X}^{W} & =\sum_{i=a-1}^{a+1} \sum_{j=b-2}^{b-1}|X(i, j)-X(i, j+1)| \\
D_{X}^{E} & =\sum_{i=a-1}^{i=a+1} \sum_{\substack{j=b \\
j=1}}^{b+1}|X(i, j)-X(i, j+1)| \\
D_{X}^{N} & =\sum_{\substack{a-1 \\
b+1}}^{i=a-2} \sum_{j=b-1}^{a+1}|X(i, j)-X(i+1, j)| \\
D_{X}^{S} & =\sum_{i=a}^{b+1} \sum_{j=b-1}^{b+1}|X(i, j)-X(i+1, j)|
\end{aligned}
$$

where $X(i, j)$ is Bayer-patterned CFA at position $(i, j)$, and $(a, b)$ is the position of the central missing color component in the local sliding window. $D_{X}^{W}$ and $D_{X}^{E}$ are the absolute color gradients of $G_{12}$ and $G_{13}$ in the horizontal direction, and $\mathrm{D}_{\mathrm{X}}^{\mathrm{N}}$ and $\mathrm{D}_{\mathrm{X}}^{\mathrm{S}}$ are the absolute color gradients of $\mathrm{G}_{9}$ and $\mathrm{G}_{16}$ in the vertical direction.

The inverse items of absolute color difference are used as weight factors to adjust for the contribution of each $\kappa R$ value according to their spectral correlation with the central missing color component. The weight allocated to each $\kappa \mathrm{R}$ is listed as follows:

$$
\begin{array}{ll}
w_{R 5}^{W}=\frac{1}{D_{X}^{W}}, & w_{R 5}^{E}=\frac{1}{D_{X}^{E}} \\
w_{R 5}^{N}=\frac{1}{D_{X}^{N}}, & w_{R 5}^{S}=\frac{1}{D_{X}^{S}}
\end{array}
$$

Then, the generalized color difference $\kappa_{R 5}$ of the central missing green component and $R_{5}$ are estimated using the surrounding $\kappa_{R}$ value and its corresponding weight by the following equation:

$$
\begin{gathered}
\kappa_{\mathrm{R} 5}=\left(\kappa_{\mathrm{R} 9} * w_{R 5}^{N}+\kappa_{\mathrm{R} 16} * w_{R 5}^{S}+\kappa_{\mathrm{R} 12} * w_{R 5}^{W}\right. \\
\left.+\kappa_{\mathrm{R} 13} * w_{R 5}^{E}\right) /\left(w_{R 5}^{N}+w_{R 5}^{S}+w_{R 5}^{W}\right. \\
\left.+w_{R 5}^{E}\right)
\end{gathered}
$$

Finally, the missing green component $\widetilde{\mathrm{G}}_{\mathrm{R} 5}$ is interpolated at the position of R5 as:

$$
\tilde{G}_{R 5}=R_{5}+\kappa_{\mathrm{R} 5}
$$

\section{B. Green Channel Refinement}

Once the missing green component is populated, it can be used to determine the interpolation direction in the refinement step. In the refinement step, every preinterpolated color component is refined by combining the estimates obtained from its four interpolation directions by exploiting the spectral correlation among the neighboring pixels along that direction. Utilizing the color difference between the $R$ and $G$ channels, $\widetilde{G}_{\mathrm{R} 5}$ can be estimated along the four directions. Referring to Fig. 1, $\widetilde{\mathrm{G}}_{\mathrm{R} 5}$ is estimated as $\widetilde{\mathrm{G}}_{\mathrm{R} 5}^{\mathrm{N}}, \widetilde{\mathrm{G}}_{\mathrm{R} 5}^{\mathrm{S}}, \widetilde{\mathrm{G}}_{\mathrm{R} 5}^{\mathrm{W}}$, and $\widetilde{\mathrm{G}}_{\mathrm{R} 5}^{\mathrm{E}}$ in these four directions as:

$$
\begin{aligned}
& \tilde{G}_{R 5}^{N}=G_{9}+0.5 *\left(R_{5}-R_{2}\right) \\
& \tilde{G}_{R 5}^{s}=G_{16}+0.5 *\left(R_{5}-R_{8}\right) \\
& \tilde{G}_{R 5}^{W}=G_{12}+0.5 *\left(R_{5}-R_{4}\right) \\
& \tilde{G}_{R 5}^{E}=G_{12}+\underset{\sim}{0.5} *\left(R_{5}-R_{6}\right)
\end{aligned}
$$

For better estimation of $\widetilde{\mathrm{G}}_{\mathrm{R} 5}$, we assign each estimate with an appropriate weight using the pre-interpolated green channel, and the directional gradients of R5 along the four directions are calculated by:

$$
\begin{aligned}
& \Delta^{N}=\left|G_{5}-\tilde{G}_{B 6}\right|+\left|\tilde{G}_{R 2}-G_{9}\right|+\left|G_{6}-\tilde{G}_{B 7}\right| \\
& \quad+\left|\tilde{G}_{B 6}-G_{12}\right|+\left|G_{9}-\tilde{G}_{R 5}\right|+\left|\tilde{G}_{B 7}-G_{13}\right|+\varepsilon \\
& \Delta^{S}=\left|G_{12}-\tilde{G}_{B 10}\right|+\left|\tilde{G}_{R 5}-G_{16}\right|+\left|G_{13}-\tilde{G}_{B 11}\right| \\
& \quad+\left|\tilde{G}_{B 10}-G_{19}\right|+\left|G_{16}-\tilde{G}_{R 8}\right|+\left|\tilde{G}_{B 11}-G_{20}\right|+\varepsilon \\
& \Delta^{W}=\left|G_{8}-\tilde{G}_{B 6}\right|+\left|\tilde{G}_{R 4}-G_{12}\right|+\left|G_{15}-\tilde{G}_{B 10}\right| \\
& \quad+\left|\tilde{G}_{B 6}-G_{9}\right|+\left|G_{12}-\tilde{G}_{R 5}\right|+\left|\tilde{G}_{B 10}-G_{16}\right|+\varepsilon \\
& \Delta^{E}=\left|G_{9}-\tilde{G}_{B 7}\right|+\left|\tilde{G}_{R 5}-G_{13}\right|+\left|G_{16}-\tilde{G}_{B 11}\right| \\
& \quad+\left|\tilde{G}_{B 7}-G_{10}\right|+\left|G_{13}-\tilde{G}_{R 6}\right|+\left|\tilde{G}_{B 11}-G_{17}\right|+\varepsilon
\end{aligned}
$$


Where, $\varepsilon$ is a small positive factor to avoid the gradient being zero. The interpolation direction of $\mathrm{R}_{5}$ is determined by the directional gradients according to the distribution situation of the four directional gradients of $\mathrm{R}_{5}: \Delta^{\mathrm{N}}, \Delta^{\mathrm{S}}, \Delta^{\mathrm{W}}$, and $\Delta^{\mathrm{E}}$. The final interpolation of $\widetilde{\mathrm{G}}_{\mathrm{R} 5}$ can be classified as one of three situations.

If $\alpha\left(\Delta^{\mathrm{W}}+\Delta^{\mathrm{E}}\right)<\left(\Delta^{\mathrm{N}}+\Delta^{\mathrm{S}}\right)$, the interpolation direction is determined to be horizontal, and the interpolation is only applied in the west and east directions.

If $\alpha\left(\Delta^{\mathrm{N}}+\Delta^{\mathrm{S}}\right)<\left(\Delta^{\mathrm{W}}+\Delta^{\mathrm{E}}\right)$, the interpolation direction is determined to be vertical, and the interpolation is only applied in the north and south directions.

Otherwise, the interpolation direction is undefined, and the interpolation is applied along all four directions. Here, the coefficient $\alpha(\alpha \geq 1)$ is used as a constraint factor to judge the interpolation direction.

The inverse of the directional gradients are used as the weight factors to adjust the joint contribution of estimation along the four interpolation directions similarly to the preinterpolation step. They are represented as:

$$
\eta^{N}=\frac{1}{\Delta^{N}}, \quad \eta^{S}=\frac{1}{\Delta^{S}}, \quad \eta^{W}=\frac{1}{\Delta^{W}} \quad, \eta^{E}=\frac{1}{\Delta^{E}}
$$

The interpolation equations are given according to the three determined directions. For horizontal interpolation, the estimations of $\widetilde{\mathrm{G}}_{5}^{\mathrm{W}}$ and $\widetilde{\mathrm{G}}_{5}^{\mathrm{E}}$ in the horizontal direction are used, and the weighting factors of $\eta^{\mathrm{W}}$ and $\eta^{\mathrm{E}}$ are involved in order to adjust the interpolation performance. For horizontal interpolation, the normalized interpolation equation is given by:

$$
\tilde{G}_{R 5}=\frac{\tilde{G}_{R 5}^{W} * \eta^{W}+\tilde{G}_{R 5}^{E} * \eta^{E}}{\eta^{W}+\eta^{E}}
$$

Similarly, for vertical interpolation, the normalized interpolation equation is defined as:

$$
\tilde{G}_{R 5}=\frac{\tilde{G}_{R 5}^{N} * \eta^{N}+\tilde{G}_{R 5}^{s} * \eta^{s}}{\eta^{N}+\eta^{s}}
$$

For the case where the interpolation direction is undefined, the interpolation is estimated along the four directions in order to avoid interpolation error. In other words, we use the joint contribution of all the preestimations $\widetilde{\mathrm{G}}_{\mathrm{R} 5}^{\mathrm{N}}, \widetilde{\mathrm{G}}_{\mathrm{R} 5}^{\mathrm{S}}, \widetilde{\mathrm{G}}_{\mathrm{R} 5}^{\mathrm{W}}$, and $\widetilde{\mathrm{G}}_{\mathrm{R} 5}^{\mathrm{E}}$ in four directions to guarantee the accuracy of interpolation with the weighting factors of $\eta^{\mathrm{N}}, \eta^{\mathrm{S}}, \eta^{\mathrm{W}}$, and $\eta^{\mathrm{E}}$. The normalized interpolation equation is defined as:

$$
=\frac{\tilde{G}_{R 5}^{n} * \eta^{N}+\tilde{G}_{R 5}^{s} * \eta^{S}+\tilde{G}_{R 5}^{w} * \eta^{W}+\tilde{G}_{R 5}^{e} * \eta^{E}}{\eta^{N}+\eta^{S}+\eta^{W}+\eta^{E}}
$$

By applying the above procedures to all red and blue positions, we can refine the green plane.

\section{Interpolating the Missing Red and Blue Components}

From the Bayer CFA samples, the green pixels are initially interpolated by the proposed method. Since the red, green, and blue planes are highly correlated, the interpolation process for $\mathrm{R}$ and $\mathrm{B}$ uses their color difference planes to avoid color mis-registration problems. First, the color difference planes $\delta_{\mathrm{RG}}$ and $\delta_{\mathrm{BG}}$ are calculated by Eq. (12).

$$
\delta_{\mathrm{RG}}=\mathrm{R}-\mathrm{G}, \quad \delta_{\mathrm{BG}}=\mathrm{B}-\mathrm{G}
$$

Thus, red and blue pixels can be reconstructed by Eq. (13) as follows:

$$
\mathrm{R}=\mathrm{G}+\delta_{\mathrm{RG}}, \quad \mathrm{B}=\mathrm{G}+\delta_{\mathrm{BG}}
$$

Specifically, the color difference planes are calculated under two conditions: the missing red and blue components at green CFA sampling positions and the missing blue (or red) components at red (or blue) sampling positions. Different neighboring pixels are used to interpolate the missing red and blue pixels according to the position condition.

In order to reduce the interpolation artifacts, a refinement scheme processes the interpolated green samples $G$ first to enhance the interpolation performance, and based on the refined green plane, it performs a refinement of the interpolated red and blue samples. More details on this refinement scheme can be found in $[7,12]$.

\section{EXPERIMENTAL RESULTS}

In this section, the proposed local adaptive directional interpolation algorithm (LADI) is evaluated both objectively and subjectively, and compared with various Demosaicking methods. The first 18 digital color images from the Kodak image dataset and were used to generate a set of testing images [15]. To conduct the experiments, we first implemented the mosaicking procedure using a Bayer color filter array on the target testing images, and then applied different Demosaicking methods to reconstruct the whole three-color-channel demosaicked image. Finally, we compared LADI with the DL, LSLCD, EDEP, AFD, and ESF methods. In addition, a refinement non-embedded LADI (labeled $\mathrm{LADI}_{\mathrm{N}}$ ) was also listed to determine the improvement in the embedded refinement method in LADI. To validate the proposed algorithm we conducted simulations using MATLAB 2009a on a Intel(R) Core(TM) i5 CPU M460 @2.53GHZ processor.

Table I shows the color peak signal-to-noise ratio (CPSNR) for objective comparison. It can be seen from Table I that our proposed method gave the highest average CPSNR value, and ESF and DL were the second and the third best of the compared methods. On the other hand, LSLCD showed the worst objective quality in the comparison. It is obvious that after refinement, LADI has a much higher CPSNR value than $\mathrm{LADI}_{\mathrm{N}}$.

Table II shows the objective image quality with the index 
of zipper effect ratio (ZER) [13]. In terms of ZER, the proposed LADI method gives the best performance with the least severe zipper effect, followed by ESF, which had the second least serious zipper effect. Although AFD had the lowest ZER value in many test images (the third best performance in average ZER metric), it did not have any advantage in terms of average ZER due to the lack of robustness and reliability for all images. In comparison, it can be intuitively observed that LADI outperformed LADI ${ }_{N}$ due to the efficiency of the refinement processing.

From the comparisons of the three objective evaluations, LADI showed competitive performance among all the methods tested, and its interpolation of various test images was accurate and robust. It should be noted that all of the measures in our experiments were computed after removing a ten-pixel-wide boundary around the border of the image.

For subjective evaluation, we used images \#1, \#15 from the Kodak dataset for subjective performance evaluation. Zoomed-in portions of demosaicked images are presented in Figs. 2(a) and Figs.3 (a). In Figs. 2, the counterpart images from the compared Demosaicking methods are shown to demonstrate artifact abilities blocking along the intensive edges of the window shades. The demosaicked image from LADI showed clear edges, just like the original figure. LADI caused the fewest color artifacts compared to other methods, as seen in Fig. 2(i). It is noteworthy that even without refinement, $\mathrm{LADI}_{\mathrm{N}}$ caused fewer interpolation artifacts than other methods, which can be seen in Fig. 2(g). A similar comparison of texture-preserving ability using image \#15 is shown in Figs. 3. In Fig. 3(a), the windows with blinds have an intensive and texture-like narrow edge. Due to the wellexploited inter-channel correlation, the edge direction is well estimated. Thus, even with this narrow, short edge, our proposed methods $\mathrm{LADI}_{\mathrm{N}}$ and LADI, can recover the edge with inconspicuous color artifacts, as shown in Fig. 3(g, h). The other methods showed more or fewer color artifacts and suffered distortions in the edge direction to a variable degree, which can be seen in Figs. 3(b-f).

$\begin{array}{llllllll}B_{1} & G_{1} & B_{2} & G_{2} & B_{3} & G_{3} & B_{4} \\ G_{4} & R_{1} & G_{5} & R_{2} & G_{6} & R_{3} & G_{7} \\ B_{5} & G_{8} & B_{6} & G_{9} & B_{7} & G_{10} & B_{8} \\ G_{11} & R_{4} & G_{12} & R_{5} & G_{13} & R_{6} & G_{14} \\ B_{9} & G_{15} & B_{10} & G_{16} & B_{11} & G_{17} & B_{12} \\ G_{18} & R_{7} & G_{19} & R_{8} & G_{20} & R_{9} & G_{21} \\ B_{13} & G_{22} & B_{14} & G_{23} & B_{15} & G_{24} & B_{16}\end{array}$

Figure 1. A $7 \times 7$ Bayer CFA block.

\section{CONCLUSIONS}

In this paper, we proposed an efficient Demosaicking algorithm that applies a gradient inverse weighted interpolation method along the interpolation direction as determined by the distribution of the directional gradient. The results showed that our method can determine the interpolation direction accurately. By using the refinement method within the same green channel, artifacts can be avoided. Consequently, our proposed interpolation method has advantages for preserving smooth edges and details.

\section{ACKNOWLEDGMENT}

This work was sponsored by NUPTSF (Grant No.NY213087).

\section{REFERENCES}

[1] B. E. Bayer, “Color imaging array,” U.S. Patent 3971 065, July 1976.

[2] H. J. Trussell and R. E. Hartwig, "Mathematics for demosaicking," IEEE Trans. Image Processing, vol. 11, no. 4, pp. 485-492, Apr. 2002.

[3] S. C. Pei and I. K. Tam, "Effective color interpolation in CCD color filter arrays using signal correlation,” IEEE Trans. Circuits and Systems for Video Technology, vol.13, no. 6, pp.503-513, Jun. 2003.

[4] X. Li, “Demosaicing by successive approximation,” IEEE Trans. Image Processing, vol. 14, no. 3, pp. 370-379, Mar. 2005.

[5] L. Zhang and $\mathrm{X}$. Wu, "Color demosaicking via directional linear minimum mean square-error estimation,” IEEE Trans. Image Processing, vol. 14, no. 12, pp. 2167-2178, Dec. 2005.

[6] B. Leung, G. Jeon, and E. Dubois, "Least-squares luma-chroma demultiplexing algorithm for Bayer demosaicking,” IEEE Trans. Image Processing, vol. 20, no. 7, pp. 1885-1894, Jul. 2011.

[7] W. J. Chen and P. Y.Chang, "Effective demosaicing algorithm based on edge property for color filter arrays,” Digital Signal Processing, vol. 22, no. 1, pp. 163-169, 2012.

[8] N. X. Lian, L. Chang, Y. -P. Tan, and V. Zagorodnov, “Adaptive filtering for color filter array demosaicking,” IEEE Trans. Image Processing, vol. 16, no. 10, pp. 2515-2525, Oct. 2007.

[9] I. Pekkucuksen and Y. Altunbasak, "Edge strength filter based color filter array interpolation,” IEEE Trans. Image Processing, vol. 21, no. 1, pp. 393-397, Jan. 2012.

[10] W. Lu and Y. Tan, "Color filter array demosaicking: new method and performance measures,” IEEE Trans. Image Processing, vol. 12, no. 10, pp. 1194-1210, 2003.

[11] K. H. Chung and Y. H. Chan. "Color demosaicing using variance of color differences,” IEEE Trans. Image Processing, vol. 15, no. 10, pp. 2944-2955, Oct. 2006.

[12] C. Y. Tsai and K. T. Song, “A new edge-adaptive demosaicking algorithm for color filter arrays,” Image and Vision Computing, vol. 25, no. 9, pp. 1495-1508, Sept. 2007.

[13] A. Buades, B. Coll, J.-M. Morel, and C. Sbert, "Self-similarity driven color demosaicking,” IEEE Trans.Image Processing, vol. 18, no. 6, pp. 1192-1202, June 2009.

[14] X. Li, B. Gunturk, and L. Zhang, "Image demosaicing: a systematic survey,” in Proc. of SPIE, vol. 6822, pp. 68221J, 2008.

[15] Kodak color image dataset, http://r0k.us/graphics/kodak/ 


\section{Alunts \\ PRESS}

Advances in Computer Science Research, (ACSR), volume 52

TABLE I. TABLE CPSNR COMPARISON (IN DB) OF DIFFERENT DEMOSAICKING SCHEMES FOR KODAK IMAGE DATASET

\begin{tabular}{|c|c|c|c|c|c|c|c|c|}
\hline Image & DL & LSLCD & EDEP & AFD & ESF & LADI $_{\mathbf{N}}$ & LADI & Rank \\
\hline 1 & 38.396 & 39.367 & 38.434 & 37.432 & $\mathbf{3 9 . 8 0 1}$ & 37.624 & 39.548 & 2 \\
\hline 2 & $\mathbf{4 0 . 8 4 9}$ & 39.533 & 39.976 & 40.629 & 40.738 & 39.857 & 40.106 & 4 \\
\hline 3 & 42.552 & 40.498 & 42.668 & 42.516 & 42.348 & 42.441 & $\mathbf{4 2 . 9 4 3}$ & 1 \\
\hline 4 & 40.436 & 39.514 & 40.448 & 40.418 & 39.95 & 40.528 & $\mathbf{4 0 . 6 9 4}$ & 1 \\
\hline 5 & 37.966 & 36.788 & 38.058 & 37.903 & 37.418 & 37.274 & $\mathbf{3 8 . 4 6 8}$ & 1 \\
\hline 6 & 40.104 & 40.132 & 39.588 & 37.859 & $\mathbf{4 1 . 0 6 4}$ & 38.145 & 40.253 & 2 \\
\hline 7 & 42.316 & 40.814 & 42.268 & $\mathbf{4 2 . 8 2 3}$ & 42.083 & 42.568 & 42.651 & 2 \\
\hline 8 & 35.978 & 35.744 & 35.862 & 35.096 & $\mathbf{3 7 . 1 0 5}$ & 35.424 & 36.537 & 2 \\
\hline 9 & 42.972 & 41.335 & 42.677 & 42.615 & 42.886 & 42.127 & $\mathbf{4 3 . 1 1 5}$ & 1 \\
\hline 10 & 42.563 & 41.759 & 42.522 & 42.62 & 42.457 & 41.864 & $\mathbf{4 2 . 7 5 8}$ & 1 \\
\hline 11 & 39.934 & 39.602 & 39.712 & 39.163 & $\mathbf{4 0 . 5 5 4}$ & 38.379 & 40.057 & 2 \\
\hline 12 & 43.368 & 42.732 & 43.283 & 42.586 & 43.658 & 42.539 & $\mathbf{4 3 . 6 9 8}$ & 1 \\
\hline 13 & 34.712 & 35.888 & 35.009 & 33.655 & $\mathbf{3 6 . 0 0 8}$ & 34.543 & 35.49 & 4 \\
\hline 14 & 36.781 & 34.364 & 36.204 & $\mathbf{3 6 . 9 2 3}$ & 35.844 & 36.784 & 36.538 & 4 \\
\hline 15 & $\mathbf{3 9 . 7 9 9}$ & 39.149 & 39.704 & 39.78 & 39.186 & 39.16 & 39.698 & 4 \\
\hline 16 & 43.667 & 43.555 & 42.933 & 40.962 & $\mathbf{4 4 . 2 2 6}$ & 42.005 & 43.954 & 2 \\
\hline 17 & 41.574 & 41.204 & 41.596 & 41.127 & $\mathbf{4 1 . 6 7}$ & 40.207 & 41.503 & 4 \\
\hline 18 & 37.777 & 37.533 & 37.803 & 37.405 & $\mathbf{3 7 . 9 8 3}$ & 36.937 & 37.925 & 2 \\
\hline Avg. & 40.097 & 39.417 & 39.930 & 39.528 & 40.277 & 39.356 & $\mathbf{4 0 . 3 3 0}$ & 1 \\
\hline
\end{tabular}

TABLE II.TABLE ZER COMPARISON OF DIFFERENT DEMOSAICKING SCHEMES FOR THE KODAK IMAGE DATASET

\begin{tabular}{|c|c|c|c|c|c|c|c|c|}
\hline Image & DL & LSLCD & EDEP & AFD & ESF & LADI $_{\mathbf{N}}$ & LADI & Rank \\
\hline 1 & 1.721 & 2.178 & 1.807 & 1.835 & $\mathbf{1 . 3 5 1}$ & 1.936 & 1.481 & 2 \\
\hline 2 & 1.098 & 2.634 & 1.288 & 1.018 & 1.248 & $\mathbf{1 . 0 9 7}$ & 1.296 & 6 \\
\hline 3 & 0.605 & 1.363 & 0.641 & 0.594 & 0.566 & 0.643 & $\mathbf{0 . 5 2 7}$ & 1 \\
\hline 4 & 0.887 & 1.267 & 1.032 & $\mathbf{0 . 8 2 5}$ & 0.924 & 1.126 & 0.971 & 4 \\
\hline 5 & 1.091 & 1.918 & 1.088 & 0.928 & 1.006 & 0.98 & $\mathbf{0 . 8 9 8}$ & 1 \\
\hline 6 & 0.942 & 1.361 & 1.084 & 1.244 & $\mathbf{0 . 7 5 3}$ & 1.116 & 0.919 & 2 \\
\hline 7 & 0.613 & 1.799 & 0.719 & 0.445 & 0.598 & $\mathbf{0 . 4 3 9}$ & 0.628 & 5 \\
\hline 8 & 1.876 & 2.602 & 2.298 & 1.735 & $\mathbf{1 . 5 1 5}$ & 1.748 & 1.629 & 2 \\
\hline 9 & 0.852 & 1.447 & 1.014 & $\mathbf{0 . 7 4 9}$ & 0.963 & 1.077 & 0.903 & 3 \\
\hline 10 & 0.869 & 1.45 & 0.993 & $\mathbf{0 . 6 7 9}$ & 0.888 & 0.928 & 0.893 & 4 \\
\hline 11 & 0.952 & 1.519 & 1.045 & 1.063 & $\mathbf{0 . 7 8 8}$ & 0.979 & 0.893 & 2 \\
\hline 12 & 0.774 & 1.318 & 0.897 & 0.955 & $\mathbf{0 . 6 5 1}$ & 1.006 & 0.758 & 2 \\
\hline 13 & 1.393 & 1.439 & 1.347 & 1.409 & 1.239 & 1.398 & $\mathbf{1 . 2 2 2}$ & 1 \\
\hline 14 & 1.09 & 1.574 & 1.135 & 1.178 & $\mathbf{0 . 9 6 8}$ & 1.215 & 0.985 & 2 \\
\hline 15 & 0.738 & 1.081 & 0.775 & $\mathbf{0 . 6 7 0}$ & 0.769 & 0.859 & 0.688 & 2 \\
\hline 16 & 0.729 & 1.064 & 0.909 & 1.132 & $\mathbf{0 . 5 6 3}$ & 0.867 & 0.679 & 2 \\
\hline 17 & 0.717 & 0.939 & 0.752 & $\mathbf{0 . 6 4 3}$ & 0.735 & 1.074 & 0.69 & 2 \\
\hline 18 & 1.188 & 1.398 & 1.191 & $\mathbf{1 . 0 6 4}$ & 1.255 & 1.256 & 1.135 & 2 \\
\hline Avg. & 1.0075 & 1.5751 & 1.1119 & 1.0092 & 0.9322 & 1.0969 & $\mathbf{0 . 9 5 5 3}$ & 1 \\
\hline
\end{tabular}




\section{ATLANTIS
PRESS}

Advances in Computer Science Research, (ACSR), volume 52

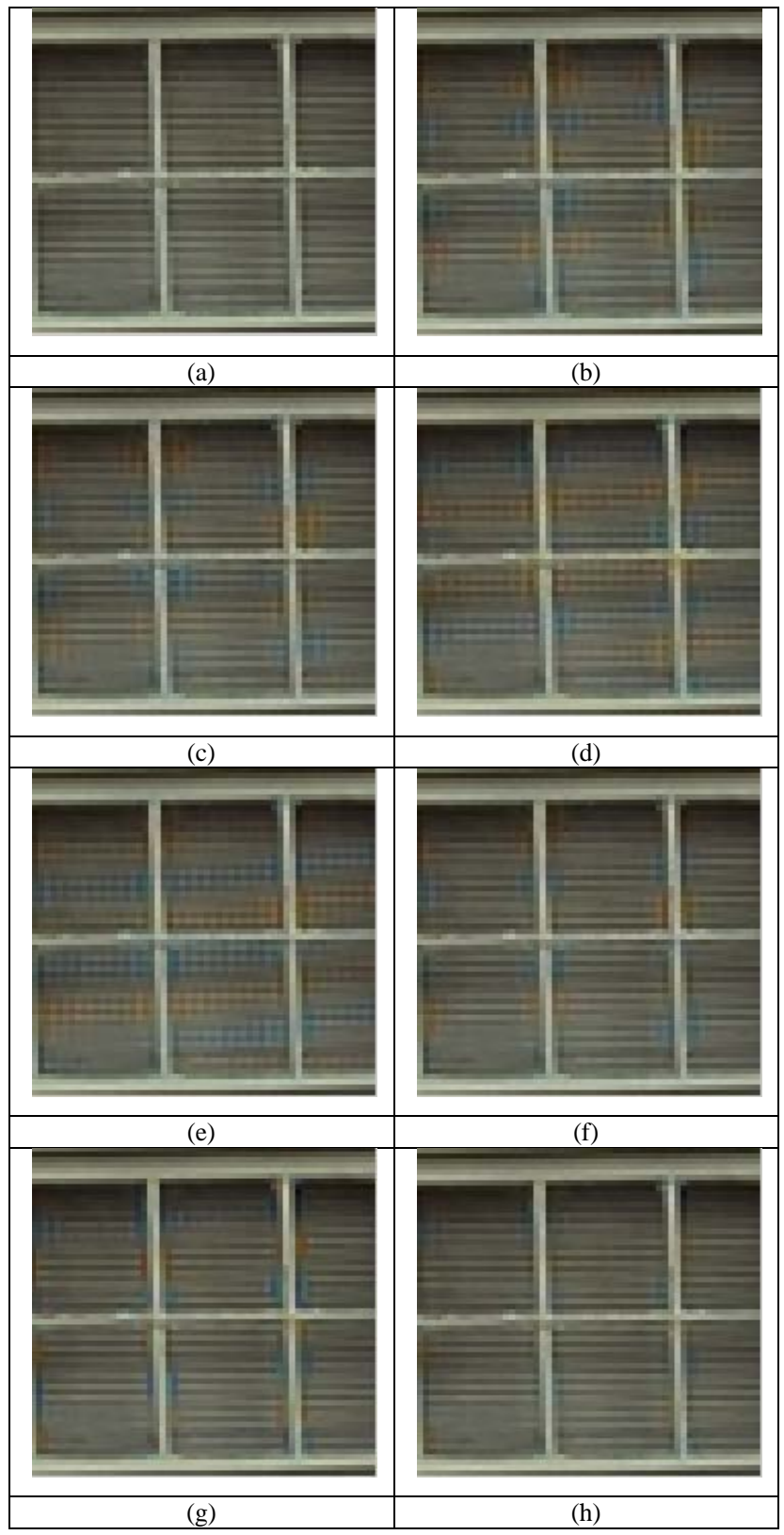

Figure 2. (a) Zoomed-in sub-image of original image \#1 and the demosaicked images by:(b) DL [5]; (c) LSLCD [6]; (d) EDEP [7]; (e) AFD [8]; (f) ESF [9]; (g) LADI ${ }_{\mathrm{N}}$ and (h) LADI.

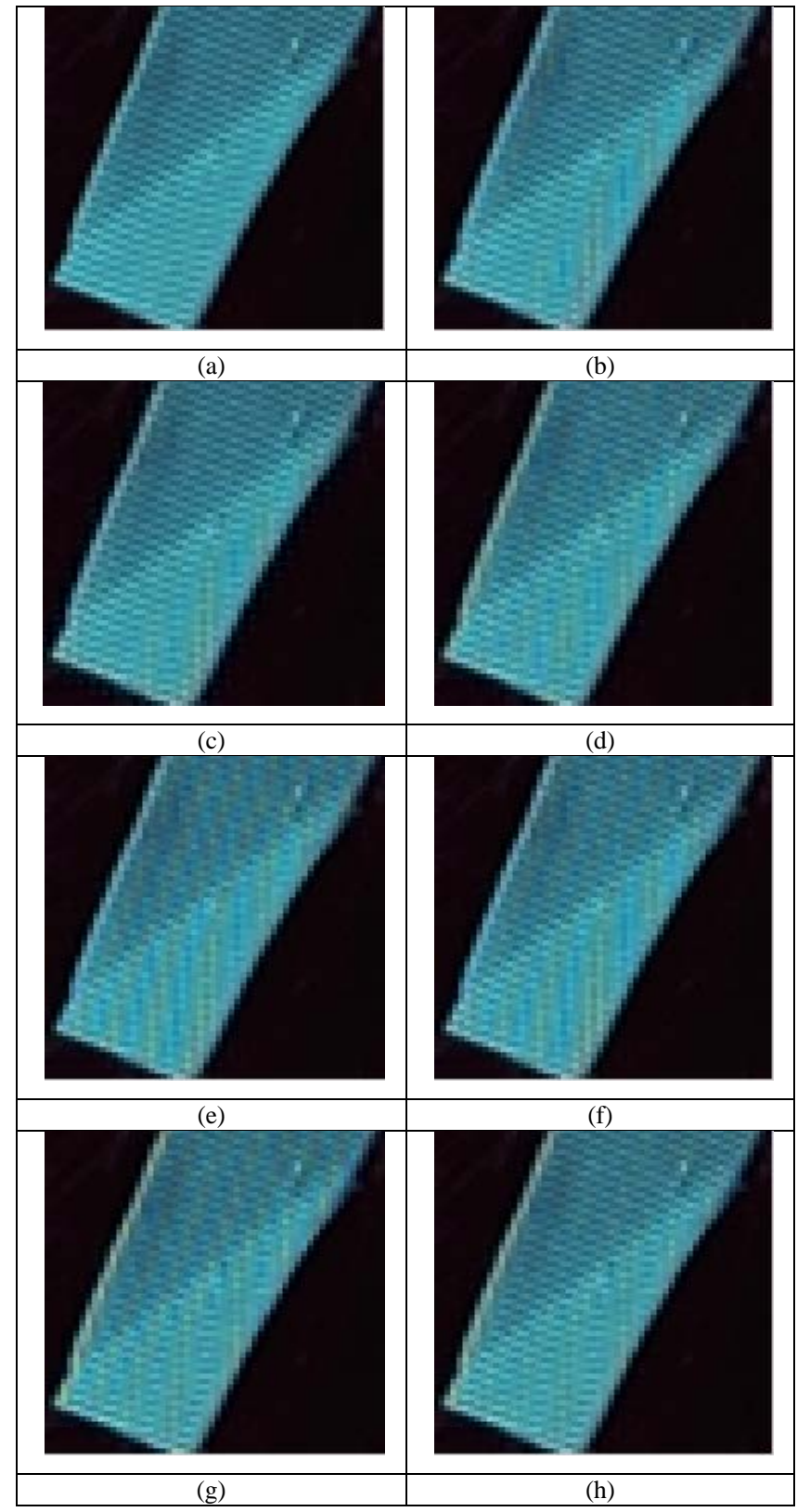

Figure 3. (a) Zoomed-in sub-image of original image \#15 and the demosaicked images by:(b) DL [5]; (c) LSLCD [6]; (d) EDEP [7]; (e) AFD [8]; (f) ESF [9]; (g) LADI ${ }_{\mathrm{N}}$ and (h) LADI. 